\title{
Light emission from direct band gap germanium containing split-interstitial defects
}

\author{
F. Murphy-Armando $\odot,{ }^{1, *}$ M. Brehm $\odot,{ }^{2, \dagger}$ P. Steindl $\odot,{ }^{1,5}$ M. T. Lusk $\odot,{ }^{3}$ T. Fromherz, ${ }^{2}$ K. Schwarz $\odot,{ }^{4}$ and P. Blaha $\odot^{4}$ \\ ${ }^{1}$ Tyndall National Institute, University College Cork, Cork T12R5CP, Ireland \\ ${ }^{2}$ Institute of Semiconductor and Solid State Physics, Johannes Kepler University Linz, Altenberger Strasse 69, A-4040 Linz, Austria \\ ${ }^{3}$ Department of Physics, Colorado School of Mines, 1500 Illinois Street, Golden, Colorado 80401, USA \\ ${ }^{4}$ Institute of Materials Chemistry, Vienna University of Technology, Getreidemarkt 9/165-TC, A-1060 Vienna, Austria \\ ${ }^{5}$ Department of Condensed Matter Physics, Faculty of Science, Masaryk University, Kotlářská 267/2, 61137 Brno, Czech Republic
}

(Received 17 December 2020; revised 26 January 2021; accepted 2 February 2021; published 25 February 2021)

\begin{abstract}
The lack of useful and cost-efficient group-IV direct band gap light emitters still presents the main bottleneck for complementary metal-oxide semiconductor-compatible short-distance data transmission, single-photon emission, and sensing based on silicon photonics. Germanium, a group-IV element like Si, is already widely used in silicon fabs. While the energy band gap of Ge is intrinsically indirect, we predict that the insertion of Ge-Ge split-[110] interstitials into crystalline Ge can open up a direct band gap transmission path. Here, we calculate from first principles the band structure and optical emission properties of $\mathrm{Ge}$, Sb, and Sn split-[110] interstitials in bulk and low-dimensional Ge at different doping concentrations. Two types of electronic states provide the light-emission enhancement below the direct band gap of Ge: a hybridized $L-\Gamma$ state at the Brillouin zone center and a conduction band of $\Delta$ band character that couples to a raised valence band along the $\Gamma-X$ direction. Majority carrier introduced to the system through doping can enhance light emission by saturation of nonradiative paths. Ge-Sn split interstitials in Ge shift the top of the valence band towards the $\Gamma-X$ direction and increase the $\Gamma$ character of the $L-\Gamma$ state, which results in a shift to longer emission wavelengths. Key spectral regions for datacom and sensing applications can be covered by applying quantum confinement in defect-enhanced Ge quantum dots for an emission wavelength shift from the midinfrared to the telecom regime.
\end{abstract}

DOI: 10.1103/PhysRevB.103.085310

\section{INTRODUCTION}

Practical monolithic solutions for direct band gap light emitters that can be readily implemented to industrial standards of Si technology are heavily sought after to push optoelectronic data transmission to the inter-and intrachip level [1-10]. Based on such emitters, the prospect of quantum applications utilizing complementary metal-oxide semiconductor-compatible single-photon sources emitting in the telecom regime would uplift the field of quantum cryptography by the power of Si-based microelectronics [11,12]. As a light source, germanium, a group-IV element such as $\mathrm{Si}$, is an obvious candidate due to its compatibility with $\mathrm{Si}$ technology and the fact that its direct band gap is only about $140 \mathrm{meV}$ larger than the indirect fundamental band gap at the $L$ points of the Brillouin zone. Recently, direct band gap emission [5-9] and even lasing at cryogenic temperatures [6] have been reported in Ge. There, uniaxial tensile strain of up to $5.9 \%$ was applied to the material, thus shifting the direct band

\footnotetext{
*philip.murphy@tyndall.ie

${ }^{\dagger}$ moritz.brehm@jku.at
}

Published by the American Physical Society under the terms of the Creative Commons Attribution 4.0 International license. Further distribution of this work must maintain attribution to the author(s) and the published article's title, journal citation, and DOI. gap at the $\Gamma$ point energetically below the $L$-point minima. Despite these promising first steps, essential bottlenecks such as efficient room-temperature operation, electrical driving at room temperature, and large-scale integration schemes are still out of reach.

Here, we propose a different pathway to direct band gap emission from a Ge crystal lattice that is supersaturated with $\mathrm{Ge}$ atoms and thus forms point defects in the form of split-[110] interstitials surrounded by crystal distortions. The Ge split-[110] interstitial is the most likely interstitial defect in Ge [13,14], and has been recently found to be the most likely to occur in Ge-ion implanted Ge [15]. Density-functional theory is employed to study the influence of the presence of these defect cores and their strained, distorted periphery onto the energy band structure and hence the light-emission properties of Ge. These defects create localized and extended states across the Brillouin zone in the electronic band structure, both influencing the light-emission properties of Ge. We argue that our approach has a high potential for applications as it is technologically feasible by combining epitaxial growth and ex situ ion implantation $[10,15,16]$.

We further expand our investigation to other elements bound to the interstitial site, such as Sb and Sn. Within concert with quantum confinement, this will allow for convenient tuning of the emission wavelength from the telecom wavelength region to the midinfrared, the fingerprint wavelength region for gas sensing. 


\section{METHODS}

\section{A. Calculation of the electronic band structure}

We performed two sets of ab initio calculations, the first to obtain the electronic band structure and the second to extract the optical emission properties. The former were conducted using the WIEN2K code $[17,18]$, which is based on the (linearized) augmented plane-wave (APW) plus local orbitals method to solve the Kohn-Sham equations [19] of densityfunctional theory (DFT). In the present work, we considered the local-density approximation (LDA) [20] since it gives better equilibrium lattice parameters than the commonly used generalized gradient approximation Perdew-Burke-Ernzerhof (PBE) [21]. We used a lattice parameter of $5.64 \AA$ and a cutoff parameter $R_{\mathrm{MT}} K_{\max }=7$, where $R_{\mathrm{MT}}$ refers to the atomic sphere radius of $2.2 \mathrm{bohr}$, and $K_{\max }$ is the plane-wave cutoff. A $4 \times 4 \times 4 k$ mesh was used, and the self-consistent field calculations and the atomic positions were fully relaxed until the forces were smaller than $1 \mathrm{mRy} / \mathrm{bohr}$. We modeled the defects by $4 \times 4 \times 4$ face-centered supercells with 129 atoms per cell. Note that cells twice as large give virtually identical results.

Once the system is relaxed, the electronic structure (energy bands, density of states, and optical properties) was calculated, including spin-orbit coupling [17] and using the modified Becke-Johnson (TB-mBJ) potential [22]. This potential provides band gaps very close to experiment, with a quality similar to that of expensive GW calculations at the cost of a standard DFT calculation [23,24]. Including spin-orbit coupling, we obtain an indirect (direct) band gap for Ge of $0.74 \mathrm{eV}(1.01 \mathrm{eV})$ at $T=0 \mathrm{~K}$. In order to even better match the experimental band gap, we reduced the $c$ parameter in the TB-mBJ functional from its self-consistent value (1.215) to 1.158 and obtained band gaps of $0.65 \mathrm{eV}(0.8 \mathrm{eV})$.

Supercell band structures are usually complicated to interpret since the backfolding of several $k$ points on the Gamma point for small Brillouin zones (BZs) (sometimes even with different symmetry) makes it difficult to disentangle the effects of the defect. One way out of this is by using a backfolding procedure, described in Refs. [25,26]. This yields a band structure in the original BZ of the defect-free material, and the corresponding spectral weight of each eigenvalue indicates the amount of original character of the bands or characterizes it as a defect state. The partial densities of states were calculated using a standard feature of APW codes, obtained by using the partial charges on the interstitial atoms as the relative weight, as explained in section III of Ref. [17].

The second set of calculations were performed using the ABINIT DFT code [27], employing HartwigesenGoedecker-Hutter (HGH) [28] norm-conserving pseudopotentials. We used this code as it already interfaces with our postprocessing code to extract the optical emission properties. The LDA [20] approximation and two sets of supercells were used: (i) $4 \times 4 \times 4$ face-centered cubic (fcc) primitive cells with a total of 129 atoms and (ii) $2 \times 2 \times 2$ cubic cells with 65 atoms for both of which we considered Ge-Ge split-[110] interstitials and Ge-Sn split[110] interstitials. Finally, the calculations were converged with a $k$ mesh of $4 \times 4 \times 4$ and a plane-wave energy cutoff of $680 \mathrm{eV}$.
To compute the alignment of the band structure of the region around the interstitial to the surrounding bulk, we utilized the methods used for alloy scattering-type impurities described in Refs. [29,30], also valid in this case. The local potentials in the vicinity of the Ge-Ge split-[110] interstitial were aligned with that of Ge bulk, and we used the latter as an energy reference to calculate the valence-band offset in the two regions. From this, we obtained a top of the valenceband offset of $V_{\text {int-bulk }}^{\mathrm{vb}}=9 \mathrm{meV}$, leading to a bottom of the conduction-band offset of $\Delta V_{\text {int-bulk }}^{\text {cb }}=-30 \mathrm{meV}$.

\section{B. Methods for the calculation of the optical properties}

The wave functions obtained in the ABINIT calculation were used to calculate the dipole matrix elements, $P_{i j}=\left\langle u_{i}|\vec{p}| u_{j}\right\rangle$, where $u_{i}$ is the $i$ th Bloch function and $\vec{p}$ the momentum operator. We computed the dipole matrix elements using the plane wave coefficients of the DFT calculated wave function,

$$
P_{i j}=\left\langle u_{i}|\vec{p}| u_{j}\right\rangle=\hbar \sum_{\boldsymbol{G}} \boldsymbol{G} C_{i}^{*}(\boldsymbol{G}) C_{j}(\boldsymbol{G}),
$$

where the summation of the plane-wave coefficients $C_{i}$ of the wave functions is over the reciprocal lattice vectors $\boldsymbol{G}$.

The photoluminescence $P L$ for free carriers due to band to band electron-hole recombination is calculated using [31]

$$
P L(\omega) \propto \omega \sum_{c, v, k} g_{k, c v} \frac{f_{k, c}\left(1-f_{k, v}\right)}{E_{k, c}-E_{k, v}} \delta\left(E_{k, c}-E_{k, v}-\omega\right),
$$

where $f_{k}$ is the Fermi distribution, which is a function of the temperature $T$ and the electron and hole chemical potentials, $E_{k, i}$ are the electronic energies of the electrons $(i=c)$ and holes $(i=v)$ at crystal momentum $k$, and $g_{k, c v}$ is the oscillator strength,

$$
g_{k, c v}=\frac{2\left|P_{k, c v}\right|^{2}}{m_{e} \hbar\left(E_{k, c}-E_{k, v}\right)},
$$

where $m_{e}$ is the electron mass and $\hbar$ is the reduced Planck constant.

To make the calculation of the photoluminescence tractable, we have made several assumptions. First, we assume that the system is in a state of quasiequilibrium before electron-hole recombination [32,33]. This means that after population inversion and before emission enough time passes for electron-phonon and electron-electron process to thermalize the electron and hole populations in their respective bands, with a uniform temperature $T$ and two chemical potentials $\mu_{v}$ and $\mu_{c}$ for the valence and conduction bands, respectively. The Fermi distributions are thus determined by the inverted electron-hole carrier population $N_{e h}$ and majority electron and hole populations $N_{n}$ and $N_{p}$, respectively:

$$
\begin{aligned}
& N_{e h}+N_{p}=\int d^{3} k f_{k, v}\left(\mu_{v}, T\right) \\
& N_{e h}+N_{n}=\int d^{3} k f_{k, c}\left(\mu_{c}, T\right) .
\end{aligned}
$$

We note here that even though the system is out of equilibrium, we can still define a plasma temperature $T$. Before the electron and hole populations equilibrate via recombination, which is on the order of nanoseconds, the electron system 
goes through two stages of equilibration via electron-electron and electron-phonon scattering. Electron-electron scattering first equilibrates the plasma temperature within each of the conduction and valence bands, reaching a common plasma temperature within femtoseconds after photoexcitation [34]. In the meantime, the system reaches two common chemical potentials for the valence and conduction bands via electronphonon scattering within $10 \mathrm{ps}$ after photoexcitation in $\mathrm{Ge}$ [35]. Therefore, in all our calculations we consider the plasma temperature equal to the lattice temperature at $T=300 \mathrm{~K}$.

A second assumption is that because the interstitial induces a direct gap, electron-phonon assisted recombination is much smaller than direct recombination, and can therefore be ignored at this stage. We also ignore excitonic effects, as their energy is on the order of 1-3 meV [36], and at the carrier populations considered here would be very screened and have little effect on the total intensity, as determined for Ge in Refs. [32,33]. Finally, Eq. (2) only includes the emission term of the PL. We assume that most of the interstitials will be close to the surface (a few $\mathrm{nm}$ ), or in small volumes in quantum dots, leaving very little time for reabsorption before the light leaves the material.

The band-structure features that give rise to strong light emission are confined to a small volume of the Brillouin zone. In photoluminescence experiments, the electrons and holes accumulate in these small volumes after energy relaxation and before recombination. Likewise, many features spread throughout the BZ. Therefore, the sum in Eq. (2) requires a very dense $k$-point grid that spans the whole $\mathrm{BZ}$ to capture the spectrum's most important emission features. To achieve such dense mesh, we built a $\mathbf{k} \cdot \mathbf{p}$ model using the dipole matrix elements between 1000 bands at the $\Gamma$ point, extending through the entire BZ. In the $\mathbf{k} \cdot \mathbf{p}$ model, the input energies above the gap were shifted up by $\Delta E=0.39 \mathrm{eV}$, where $\Delta E=E_{g}^{\mathrm{Exp}}-E_{g}^{\mathrm{LDA}}$ is the energy difference between the experimental and LDA calculated gap in pure bulk Ge. This is not a simple scissors operator, as the dispersion of the band structure is updated by the change in band-gap in the $\mathbf{k} \cdot \mathbf{p}$ model. The $\mathbf{k} \cdot \mathbf{p}$ model was used to compute the oscillator strength $g$ in Eq. (3) at any crystal momentum $\vec{k}$.

\section{RESULTS}

\section{A. Atomic structure of the defects}

The system that we are studying is peculiar in the sense that $\mathrm{Ge}$ atom supersaturation is present in the crystal, i.e., $N+X$ atoms are located in the region for which $N$ Ge atoms would be needed to form a single crystal. $X$ is then the number of atoms (Ge, $\mathrm{Sn}$, or $\mathrm{Sb}$ ) that can be experimentally incorporated, e.g., via ion implantation into the single crystal. Here, we limit $X$ to 1 for the different sizes of the theoretically assessed unit cells. Thus, for cells of 129 and 65 atoms considered here, the resulting interstitial concentrations are $3.6 \times 10^{20}$ and $7.2 \times$ $10^{20} \mathrm{~cm}^{-3}$, respectively.

In such a configuration, the defect structure with the lowest formation energy was found to be the split-[110] interstitial $[13,37]$. This structure was also found as the most likely in a previous work using a Monte Carlo quench-anneal technique starting from an amorphous supersaturated Ge 65-atom slab

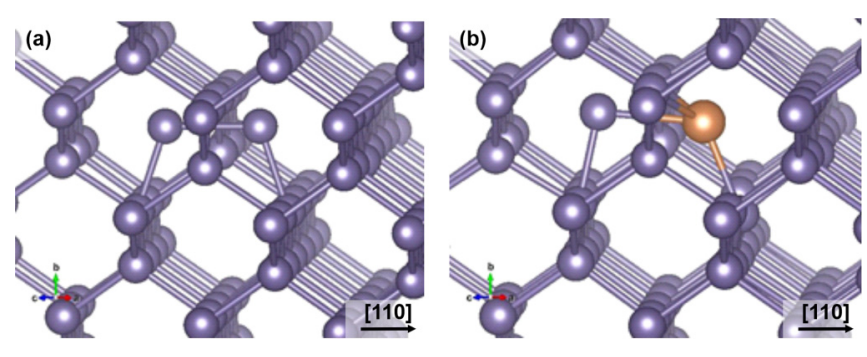

FIG. 1. Calculated atomic structure of a Ge crystal containing (a) a Ge-Ge split-[110] self-interstitial and (b) a Ge-Sb split-[110] interstitial defect. Ge atoms are indicated as violet spheres while the $\mathrm{Sb}$ atom is depicted in gold.

connected to a crystalline 65-atom slab [15]. It consists of a pair of atoms (dimer) formed by the additional atom (Ge, $\mathrm{Sn}$, or $\mathrm{Sb}$ ) and a $\mathrm{Ge}$ atom, which is moved away from its regular lattice position. In the case of a Ge-Ge split-[110] selfinterstitial, this defect is highly symmetric [13-15] [Fig. 1(a)], while for Sn-Ge or Sb-Ge split-[110] interstitials, an asymmetric structure develops; see Fig. 1(b). The distances within the dimer (Table I) are relatively long compared to the shortest connection to the regular lattice sites or the regular Ge-Ge bond distance and similar to the next-nearest neighbor (nnn) distances. Besides, some distances to further surrounding atoms are changed by a few hundredths of an $\AA$. We note that these distances are quite sensitive to the underlying lattice parameter and the computational method and, of course, to the exchange-correlation functional. For instance, in Ref [14], it is claimed that the dimer distance and the Ge next-nearest neighbor distances (Ge-nnn) are identical, while in Ref. [13], all distances turn out significantly smaller, which is probably due to a very small used lattice parameter.

\section{B. Band structure of Ge containing a split-[110] self-interstitial defect}

The calculated electronic band structure of a fcc 129Ge atom supercell containing a split-[110] self-interstitial is depicted in Fig. 2. This supercell corresponds to $4 \times 4 \times 4$ primitive fcc cells with the added perturbation of the selfinterstitial. In a $4 \times 4 \times 4 \mathrm{fcc}$ supercell, the primitive cell wave vectors at $X, 1 / 2 X$, and $L$ fold into the $\Gamma$ point in the Brillouin zone. To clarify this point, we have unfolded the supercell bands and overlaid the band structure of pure $\mathrm{Ge}$ in Fig. 3.

In Fig. 3, the calculated band structure of single-crystalline $\mathrm{Ge}$ is indicated in blue. Compared to single-crystalline $\mathrm{Ge}$, a

TABLE I. Distances (in $\AA$ ) within the dimer, the Ge-Ge nearest neighbor (nn) and next-nearest neighbor (nnn), the $\mathrm{Sn} / \mathrm{Sb}-\mathrm{Ge} \mathrm{nn}$ and $\mathrm{nnn}$ neighbor (there are $2 \mathrm{nnn}$ atoms as indicated in Fig. 1 for the $\mathrm{Sb}$ case). The bond distance in pure Ge is $2.44 \AA$.

\begin{tabular}{lccc}
\hline \hline & Dimer & Ge nn/nnn & Sn/Sb nn/nnn \\
\hline $\mathrm{Ge}$ & 2.58 & $2.43 / 2.61$ & \\
$\mathrm{Sn}$ & 2.70 & $2.43 / 2.66$ & $2.55 / 2.72$ \\
$\mathrm{Sb}$ & 2.69 & $2.44 / 2.77$ & $2.56 / 2.71$ \\
\hline \hline
\end{tabular}




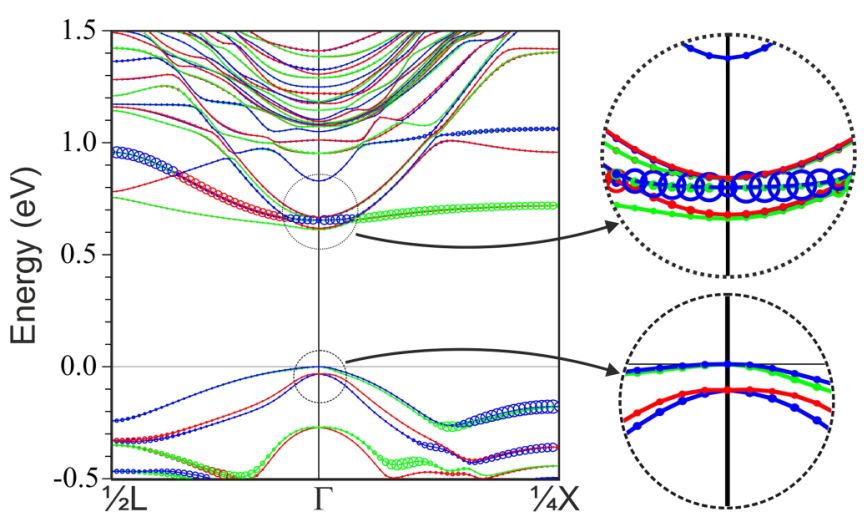

FIG. 2. Electronic band structure of a Ge interstitial in bulk Ge with the TB-mBJ ( $c=1.158)$ potential. The concentration is 1 interstitial per $128 \mathrm{Ge}$ atoms. The points labeled $L$ and $X$ on the reduced Brillouin zone correspond to the $1 / 2 L$ and $1 / 4 X$ points in the unfolded zone shown below in Fig. 3. The empty circles' size is proportional to the partial charge of the state on the interstitial atoms. The color indicates the band index.

split-[110] self-interstitial introduces several new states in the electronic band structure. In the valence band (VB), it splits the degeneracy of the light and heavy holes and introduces a degree of spin separation in the second and third valence bands (see the VB structure in Fig. 2). Additionally, raised

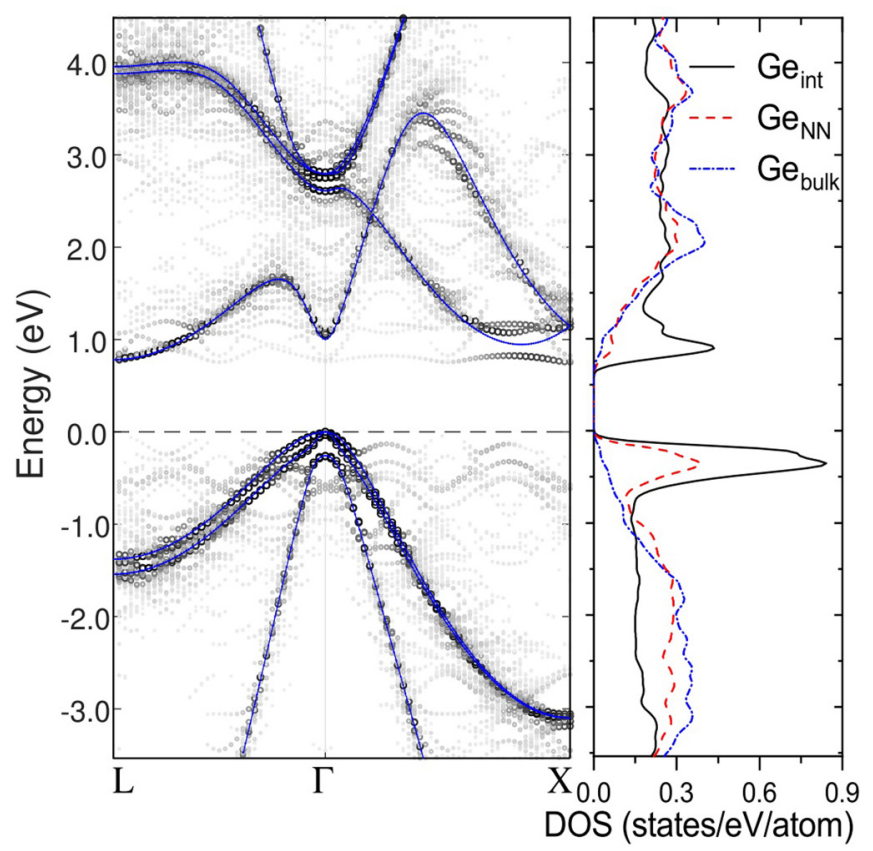

FIG. 3. Left panel: (blue) Calculated band structure of undisturbed crystalline $\mathrm{Ge}$, using the self-consistent value of the TB-mBJ potential. Gray: Unfolded electronic band structure of a Ge-Ge split[110] self-interstitial. The concentration of additional Ge atoms is 1 interstitial per $128 \mathrm{Ge}$ atoms. The darkness of the gray points indicates the proportion of overlap with a plane wave with the corresponding wave vector. Right panel: Calculated DOS in units of states per $\mathrm{eV}$ per atom for Ge bulk (blue), for the atoms contributing to the Ge split interstitial (black), and for the next-nearest Ge atoms to the split interstitial (red dashed line). valence-band local maxima at $1 / 4$ on the $\Gamma-X$ direction are introduced.

In the conduction band (CB), the defect hybridizes the four $L$ states with each other and with the $\Gamma$ direct conduction band, leading to three $L$-like states and an additional $L \Gamma$ hybrid that is close in energy to the original $L$ states in bulk Ge. We will see that the latter is of particular interest as it emits light due to its partial $\Gamma$ character and the resulting enhanced oscillator strength. The energetic ordering of these four is as follows: The lowest conduction band state is of $L$ character and hence does not couple optically to the valence band. The second lowest is the emitting $L \Gamma$ hybrid ( $10 \mathrm{meV}$ above the bottom CB state), followed by the two remaining $L$-character states (50 meV above). As evident from Fig. 3, these states have a dispersion very close to that of the original $L$ states.

We emphasize that the defect introduces another state that energetically lies between the $L \Gamma$ hybrid and the higherenergy $L$-character states, $\sim 90 \mathrm{meV}$ above the $\mathrm{CB}$ edge. Interestingly, this new state is entirely made of $X$ and $\Delta$-valley character and has a very flat in-plane $k_{x}$ and $k_{y}$ dispersion (see Figs. 2 and 3 in the $X$ direction). As seen by the size of the circles in Fig. 2, which give the proportion of the partial charge of the state on the interstitial atoms, this state is localized on the interstitial atoms. Optical coupling to the raised valence band at $1 / 4 X_{\text {orig }}$ of the original Brillouin zone for the localized state is weak but not insignificant; see the last entry in Table II.

Higher in energy than the $L, L \Gamma$, and $X$-like states, we find a band at the $\Gamma$ point that itself is $80 \%$ composed of the character of the original $\Gamma$ valley in bulk Ge $(140 \mathrm{meV}$ above the conduction-band edge), and some additional $L$ and $X$ character due to the defect. The oscillator strength of this state is high, 20.12 (see the coupling of $\Gamma_{\mathrm{VBtop}}-\Gamma_{\mathrm{CB} 6}$ in Table II), and hence a pronounced optical coupling to the valence band is present.

Thus, the split-[110] self-interstitial unusually affects the electronic structure. Rather than generating localized states, it strongly affects the energy dispersion of bulk Ge. More comparable to disordered systems, new states emerge that are a mix of the original bulk states. Those that hybridize with the optically active $\Gamma$ band will actively contribute to the photoluminescence emission. However, there exists also a degree of electronic state pinning to the defect site. Its presence is clearer in the projected density of states (DOS) per eV and atom, depicted in the right panel of Fig. 3. As compared to bulk Ge atoms (blue dashed-dotted line), the atoms at the split[110] self-interstitial site (solid black line) and its vicinity (Ge nearest-neighbors $\mathrm{Ge}_{\mathrm{NN}}$, red dashed line) induce a pronounced increase in the DOS at the band-edge minima. These pinned states are the $X$-like state in the conduction band and the raised band with a maximum at $1 / 4 X$ in the valence band.

The incorporation of Ge-Sb or Ge-Sn split-[110] interstitials into crystalline Ge that can follow, e.g., implantation of $\mathrm{Sb}$ of Sn ions, also severely affects the band structure, as can be seen in Fig. 4. While for the Ge-Sb interstitial, the VB maximum remains at the $\Gamma$ point, we find that for the $\mathrm{Ge}-\mathrm{Sn}$ split interstitial, the VB states in the $X$ direction are lifted in energy and form the clear new valence-band maximum. In both cases, the DOS close to the band gap increases in the presence of the split-[110] interstitial. We find, however, a distinct difference in the DOS between the Ge-Sb and the 
TABLE II. Oscillator strength at the Brillouin zone center and at $1 / 4 X$ for the transition of the top of the valence band ( $\Gamma_{\mathrm{VBtop}}$ ) to the first six conduction bands $\left(\Gamma_{\mathrm{CB} 1}-\Gamma_{\mathrm{CB} 6}\right.$ and $\left.1 / 4 X_{\mathrm{CB} 1}\right)$ for interstitial concentrations of 1/128 and 1/64 (i.e., one interstitial every 128 or 64 fcc Ge atoms). The element in parenthesis is that forming the interstitial.

\begin{tabular}{lccc}
\hline \hline Transition & Oscillator strength $g(\mathrm{Ge}) 1 / 128$ & Oscillator strength $(\mathrm{Sn}) 1 / 128$ & Oscillator strength $g(\mathrm{Ge}) 1 / 64$ \\
\hline$\Gamma_{\mathrm{VBtop}}-\Gamma_{\mathrm{CB} 1}$ & 0 & 0.001 & 0 \\
$\Gamma_{\mathrm{VBtop}}-\Gamma_{\mathrm{CB} 2}(L-\Gamma)$ & 1.641 & 3.02 & 3.21 \\
$\Gamma_{\mathrm{VBtop}}-\Gamma_{\mathrm{CB} 3}$ & 0.005 & 0.136 & 0.005 \\
$\Gamma_{\mathrm{VBtop}}-\Gamma_{\mathrm{CB} 4}$ & 0.010 & 0.217 & 0.014 \\
$\Gamma_{\mathrm{VBtop}}-\Gamma_{\mathrm{CB} 5}$ & 0.003 & 0.001 & 0.033 \\
$\Gamma_{\mathrm{VBtop}}-\Gamma_{\mathrm{CB} 6}$ & 20.12 & 17.6 & 14.5 \\
$1 / 4 X_{\mathrm{VBtop}}-1 / 4 X_{\mathrm{CB} 1}$ & 0.121 & 0.09 & 0.124 \\
\hline \hline
\end{tabular}

Ge-Sn defects. While for the former, the maximum DOS for the $\mathrm{Ge}$ and the $\mathrm{Sb}$ atom that contribute to the interstitial is almost at the same energetic position, this varies for the $\mathrm{Ge}-\mathrm{Sn}$ interstitial indicating that the electronic state at the Sn atom forms the VB maximum states in the $\Gamma-X$ direction.

\section{Optical properties}

This subsection explores the emission properties of the [110]-split interstitial under different configurations. Section III C 1 shows the results for the Ge-Ge [110]-split interstitial at different electron-hole populations for the lowest interstitial density considered, i.e., one interstitial atom every $128 \mathrm{Ge}$ atoms. Section III C 2 shows the effects on the light emission of changing the interstitial density. Section III C 3 explores light emission under different majority carrier concentrations. This would correspond to the case of a doped material, interstitials at different charge states, or the conse- quence of other carrier dynamic effects. Section III C 4 shows the large effects on emission produced by a $\mathrm{Sn}-\mathrm{Ge}$ interstitial. We do not consider the emission properties of a Sb-Ge interstitial explicitly, as we found that this interstitial has little effect on the band structure. The Sn-Ge interstitial would be acting as an $n$-type dopant, and thus its effects are already treated in Sec. III C 3. Finally, in Sec. III C 5 we explore what would happen to the emission wavelengths under severe quantum confinement, as would be the case in Ge quantum dots implanted with Ge ions currently under investigation [15].

\section{The Ge-Ge split-[110] self-interstitial}

We calculated the photoluminescence (PL) spectra of a $1 / 128 \mathrm{Ge}-\mathrm{Ge}$ split-[110] self-interstitial in bulk Ge for different exciton concentrations between $N_{e h}=4 \times 10^{18}$ to $5 \times$ $10^{19} \mathrm{~cm}^{-3}$, at a plasma temperature $T=300 \mathrm{~K}$, shown in Fig. 5. For these parameters, the density of excitons can be roughly compared to typical electron occupations at excitation
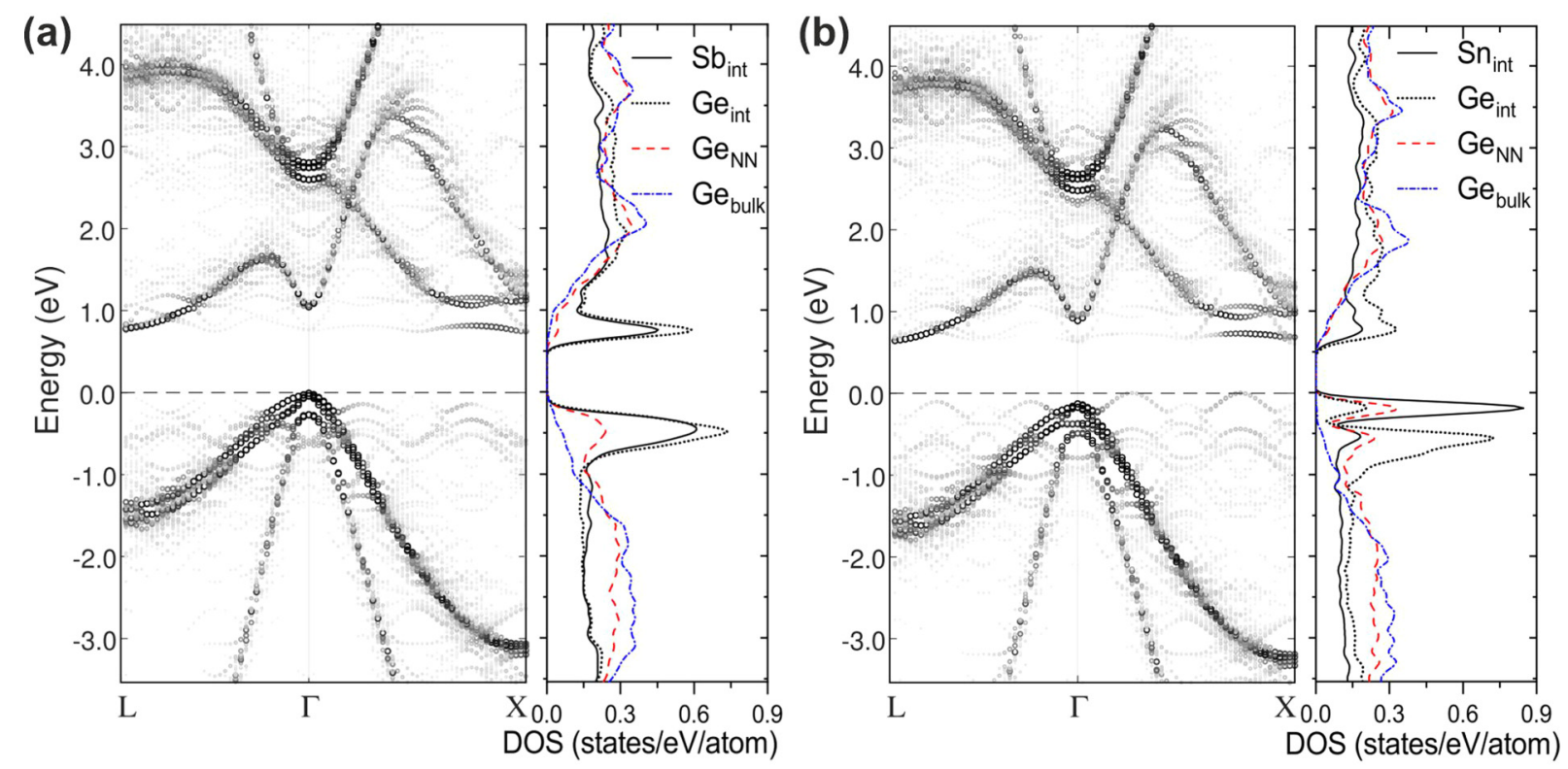

FIG. 4. Left panels: Unfolded electronic band structure (using the self-consistent value of the TB-mBJ potential) of (a) a Sb-Ge split-[110] interstitial, (b) a Ge-Sn split-[110] interstitial, both with a concentration of 1 split-interstitial per 128 Ge atoms. Notice in (b) for the Sn-Ge interstitial that the top of the VB is $1 / 4$ along the $\Gamma-X$ line, rather than at the $\Gamma$ point. The darkness of the gray points denotes the proportion of overlap with a plane wave with the corresponding wave vector. Right panel in (a) and (b): Calculated density of states in units of states per eV per atom for Ge bulk (blue), for the Ge, Sb, and Sn atoms contributing to the split interstitial (black) and for the next-nearest Ge atoms to the split interstitial (red dashed lines). 


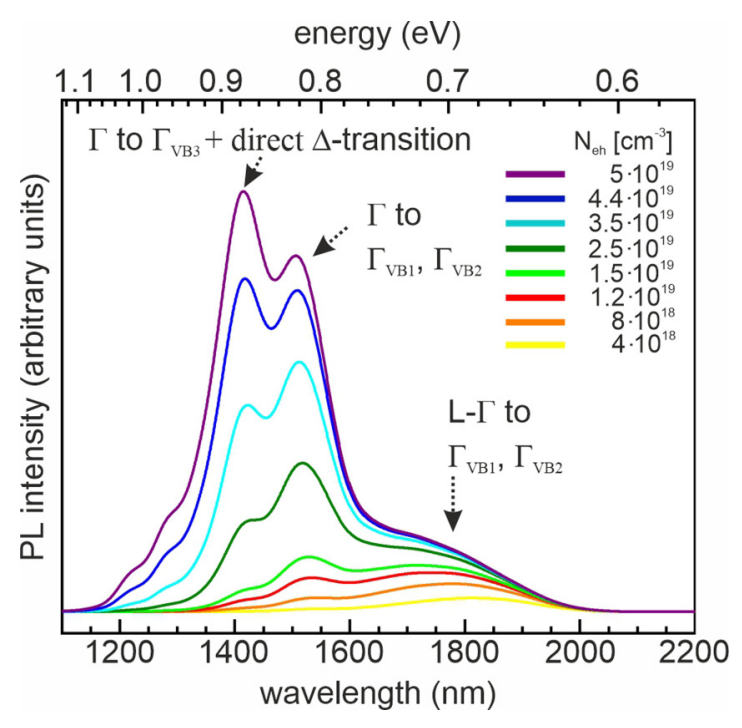

FIG. 5. Calculated PL spectrum of a Ge with $1 / 128 \mathrm{Ge}-\mathrm{Ge}$ split[110] self-interstitials for a different number of excitons, $N_{\text {eh }}$. The three distinctive peaks at 1800,1550 , and $1400 \mathrm{~nm}$ are due to the $\Gamma-L$ state to the top of the valence band, the $\Gamma_{\mathrm{cb}}$ state in pure Ge to the first and second topmost of the valence band, and the $\Gamma_{\mathrm{cb}}$ state to the third topmost valence band. Contributions from the valence-band maxima to the conduction-band pinned state at $1 / 4 X$ ( $\Delta$ direction) introduce additional intensity.

powers $P_{e x}$ used in micro-PL measurements on ensembles of $\mathrm{Ge} / \mathrm{Si}$ quantum dots and defect-enhanced quantum dots [10,38,39].

In Fig. 5, we observe three prominent peaks that dominate at different exciton densities that are all originating or at least influenced by the Ge-Ge split-[110] self-interstitial: (i) Emission from the $L-\Gamma$ mixed band in the conduction band is coupled to the top of the valence band $\Gamma_{\mathrm{VB} 1}$ and $\Gamma_{\mathrm{VB} 2}$ and occurs at a wavelength of $1750 \mathrm{~nm}$ to $1800 \mathrm{~nm}$ $(\sim 0.68 \mathrm{eV})$. (ii) At a wavelength of $\sim 1550 \mathrm{~nm}$, emission from the $\Gamma_{\mathrm{CB}}$ in pure Ge band is coupled to the top of the valence band, $\Gamma_{\mathrm{VB} 1}$ and $\Gamma_{\mathrm{VB} 2}$. (iii) The conduction band emitting at the highest energy also corresponds to the $\Gamma$ band in pure Ge coupled to the third topmost valence band at $\Gamma\left(\Gamma_{\mathrm{VB} 3}\right)$, emitting at $0.88 \mathrm{eV}(\sim 1400 \mathrm{~nm})$. This last peak also has a small contribution from a semilocalized band along the $\Delta$ line ( $\Gamma-X$ line), composed of states of $X$ character, that couple to a raised state in the valence band also along the $\Delta$ line (see Figs. 2 and 3) which emits at $1400 \mathrm{~nm}$. As we will show later, the light emission can also be affected by the concentration of interstitials and the $n$-type doping concentration.

Furthermore, the split-[110] self-interstitial breaks the degeneracy of the light-hole and heavy-hole bands. Based on our calculations, a degree of spin separation is present in the valence bands and should be observable at lower PL temperatures and high pump powers.

\section{Influence of the split-[110] interstitial density}

Such a postgrowth ion-implantation treatment naturally raises the question of the effect of the ion-implantation dose, i.e., the defect concentration, on the light-emitting properties. To obtain the effects of a different implant concentration, we

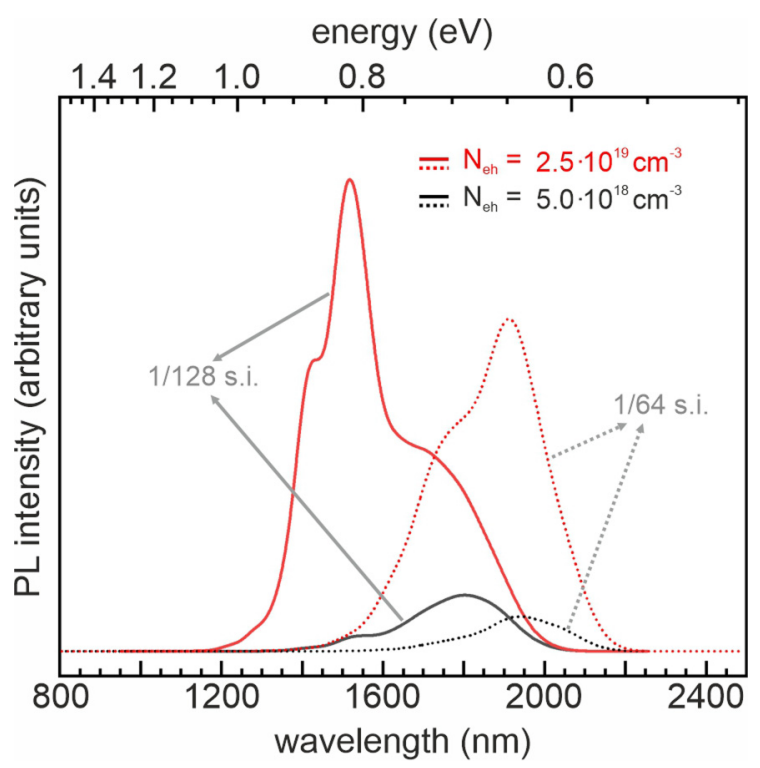

FIG. 6. Influence of the Ge-Ge split-[110] self-interstitial (s.i.) concentration on the PL for different exciton concentrations, $N_{\text {eh }}=$ $5 \times 10^{18} \mathrm{~cm}^{-3}$ (black) and at $N_{\text {eh }}=2.5 \times 10^{19} \mathrm{~cm}^{-3}$ (red). The concentration of interstitials considered increases from $1 / 128$ (solid line) to $1 / 64$ (dashed line).

calculated the band structure in two different supercells of 1/128 and 1/64 Ge-Ge interstitials per crystalline Ge atom in fcc and cubic supercells, respectively.

We find that an increase in the concentration of split-[110] self-interstitials increases the coupling of the $\mathrm{CB} \Gamma$-and $L$ states. This increases the energy splitting between hybridized $L$ states with higher interstitial concentration and the oscillator strength of the $L-\Gamma$ state (see Table II). The raised interstitial concentration results in a shift of the emission edge to longer wavelengths, as shown in Fig. 6, dotted spectra. Simultaneously, the original $\Gamma$-like conduction band in $\mathrm{Ge}$ becomes a mixed state with increasing interstitial concentration and thereby further losing its direct band character (see the reduction in oscillator strength of $\Gamma_{\mathrm{CB} 6}$ in Table II). Consequently, this slightly reduces the emission intensity of the shorter wavelength peak $(\sim 1500 \mathrm{~nm})$ with increasing interstitial concentration (Fig. 6). Also, the stronger hybridization pushes both the dark $\Gamma_{\mathrm{CB} 1}$ (with $L \mathrm{CB}$ band character) and the $L \Gamma$ hybrid state to lower energies and, thus, longer wavelengths for higher defect concentration. As more electrons populate the dark $\Gamma_{\mathrm{CB} 1}$ state, the overall PL intensity is reduced as compared to a lower concentration of interstitials.

\section{Influence of majority carriers}

In a real system, there may be an unequal number of electrons and holes present in the light-emitting material, e.g., due to doping in electrically pumped devices, different charge states of the interstitial defect, or dynamic reasons during the energy relaxation. Thus, we calculate the effect of an excess of electrons or holes on the light-emission spectrum for a system at which $5 \times 10^{18} \mathrm{~cm}^{-3}$ excitons are excited around the interstitial. The results in Fig. 7 show that an excess number of electrons or holes leads to very different spectral 


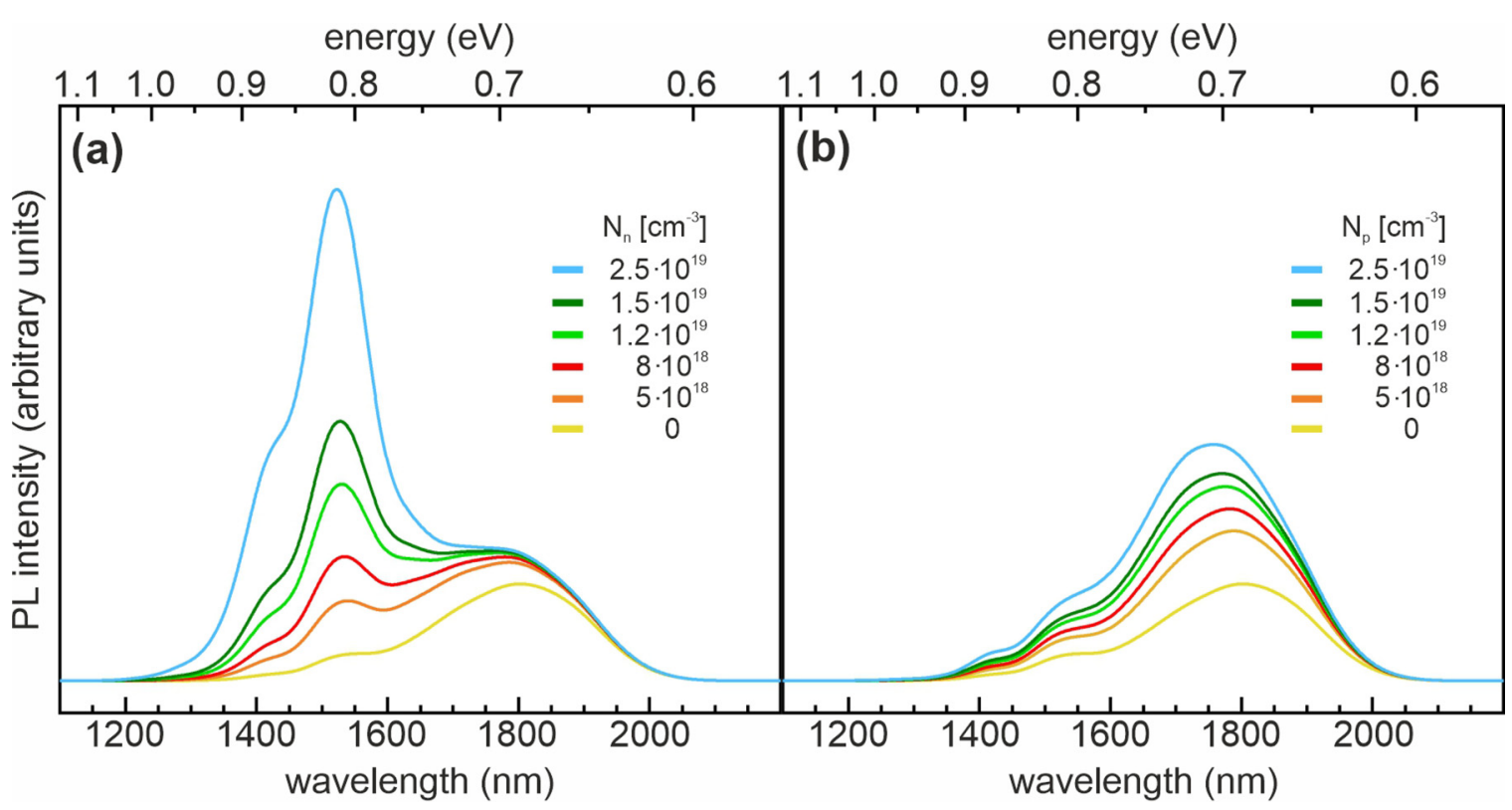

FIG. 7. Calculated photoluminescence spectra obtained for a Ge crystal containing Ge-Ge split-[110] self-interstitials and a exciton concentration of $N_{\mathrm{eh}}=5 \times 10^{18} \mathrm{~cm}^{-3}$. Additional concentrations of electrons $N_{\mathrm{n}}$ (a) and holes, $N_{\mathrm{p}}$ (b) in the range from $5 \times 10^{18}$ to $2.5 \times 10^{19} \mathrm{~cm}^{-3}$ are introduced.

emission shapes. Adding electrons leads to a distinct increase in the Ge $\Gamma_{\mathrm{cb}}$-related transition, as seen in Fig. 7(a). This increase is due to the Fermi blocking of the lower conduction bands, now filled with dopand electrons. As a consequence, additional optically pumped electrons populate the energetically higher CB states already at moderate pump rates. This results in observable emission under moderate pumping from electron states in a broad energy range from the CB edge up to these higher states. A similar emission behavior is observed in $n$-type doped strained Ge, where the $L$ band is filled with electrons, enhancing emission from the direct $\Gamma$ band [40]. An excess number of holes systematically increases the emission intensity of the transitions of the lower $\Gamma-L$ conduction-band state by increasing the states available for recombination in the valence band.

\section{Effect of Sn-Ge split-[110] interstitials}

From the above discussion of the changes in the band structure related to the Ge-Ge and Ge-Sn split-[110] interstitial, it is evident that the introduction of Sn-Ge split-[110] interstitials into Ge crystals must affect the PL-emission properties. The first striking effect of the Ge-Sn split-[110] interstitial is that the top of the valence band is not at the Brillouin-zone center but at $1 / 4 X_{\text {orig }}$ in the $\Delta$ line. With the bottom of the conduction band being at the $\Gamma$ point, the system turns into an indirectband semiconductor, and thus the PL is reduced by several times compared to the Ge-Ge split-[110] self-interstitial; see Fig. 8.

However, there are two indications that the addition of Sn-Ge split-[110] interstitials may indeed be beneficial for enhancing emission in Ge. The first is the twofold increase of the oscillator strength of the $\Gamma-L$ mixed state (see Table II) as compared to the Ge-Ge split-[110] self-interstitial. The second is the shift of the top of the VB away from the zone center to the $1 / 4 X_{\text {orig }}$ position, suggesting the localization of the exciton at the interstitial site, according to the right panel of Fig. 4(b).

As it stands, these two emission channels $\left(\Gamma_{\mathrm{VBtop}}-\Gamma_{\mathrm{CB} 2}\right.$ and $\left.1 / 4 X_{\mathrm{VBtop}}-1 / 4 X_{\mathrm{CB} 1}\right)$ are suppressed due to the indirect nature of the band gap. We can proceed as in the previous section, though, and fill the indirect bands by using doping. Additional $p$-type doping populates the top of the valence valleys at $1 / 4$

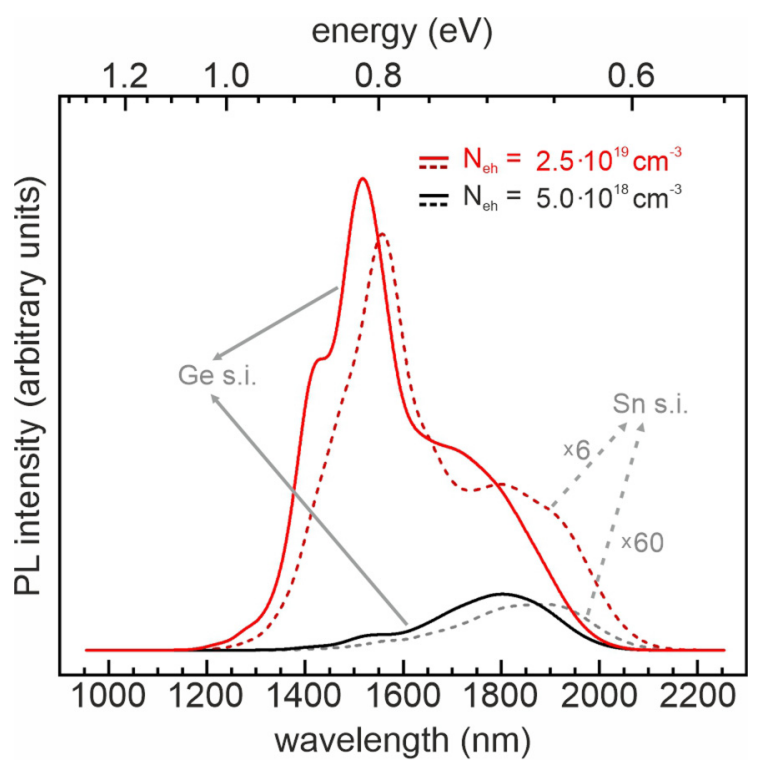

FIG. 8. Comparison of the effects of a Sn (dashed line) and Ge (solid line) split-[110] interstitial (in figure labeled as s.i.) on the calculated photoluminescence at two exciton concentrations, $N_{\text {eh }}=$ $5 \times 10^{18}$ and $2.5 \times 10^{19} \mathrm{~cm}^{-3}$. The PL emission due to the $\mathrm{Sn}-\mathrm{Ge}$ interstitial has been multiplied by a factor of 60 and 6 times, respectively, to compare to the Ge self-interstitial. 

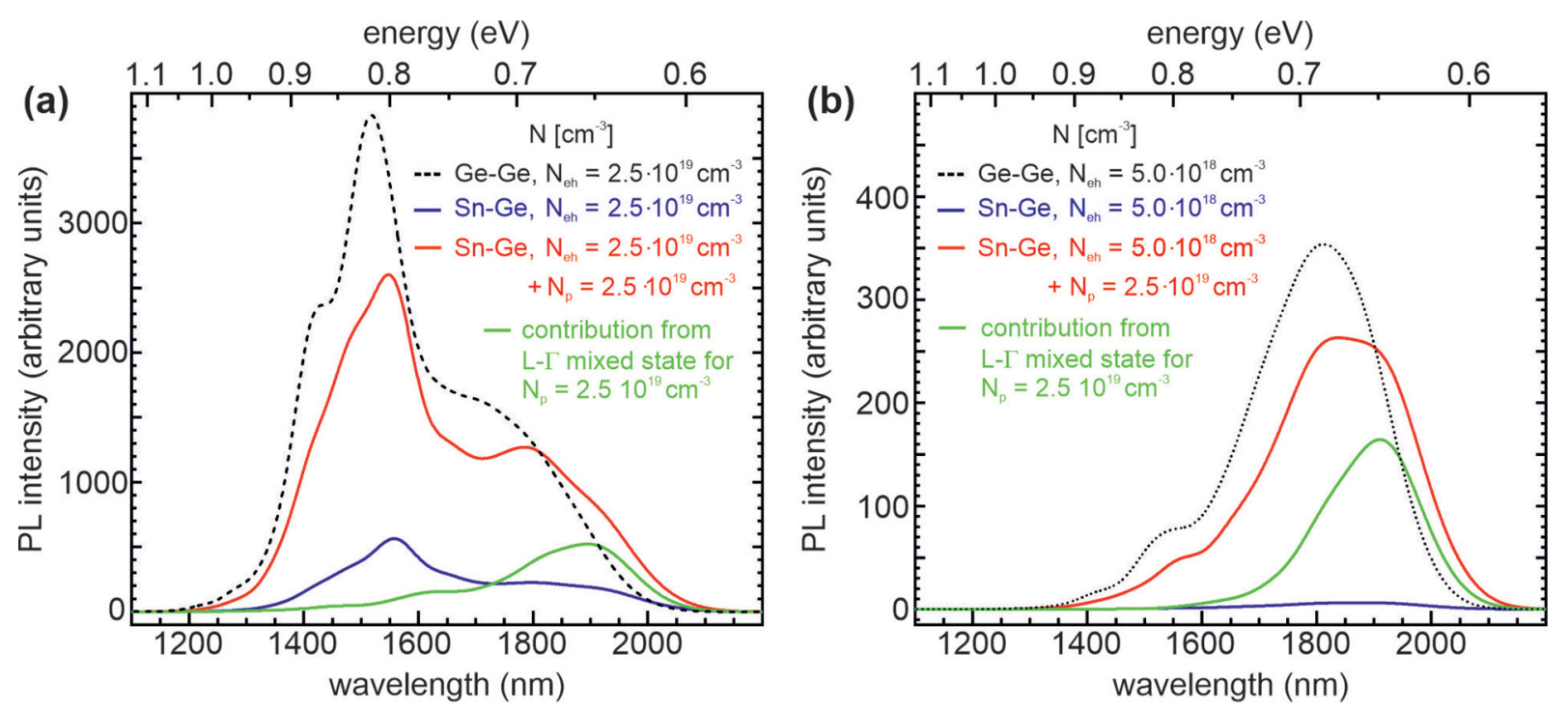

FIG. 9. (a) Calculated PL emission from Sn-Ge split-[110] interstitial at an exciton density of $2.5 \times 10^{19} \mathrm{~cm}^{-3}$ without (blue) and with additional $p$-type doping density of $2.5 \times 10^{19} \mathrm{~cm}^{-3}$ (red). The PL response from a Ge-Ge split-[110] self-interstitial without doping is depicted in black. The green line shows the contribution from the $\Gamma-L$ mixed state at $N_{\mathrm{p}}=2.5 \times 10^{19} \mathrm{~cm}^{-3}$. The contribution from the top of the valence band at $1 / 4 X_{\text {orig }}$ to the first CB at $1 / 4 X_{\text {orig }}$ to the overall PL is negligible at low $N_{\text {eh. }}$ (b) Same case as (a) except for a lower exciton concentration $N_{\text {eh }}$ of $5 \times 10^{18} \mathrm{~cm}^{-3}$.

$X_{\text {orig }}$ and enhances the emission via the direct transition at the $\Gamma$ point. $n$-type doping fills the bottom of the conduction band at the $\Gamma$ point and increases the number of carriers at the $1 / 4 X_{\text {orig }}$ conduction band, enhancing the direct transition

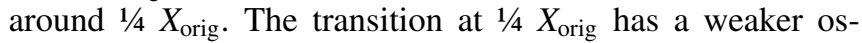
cillator strength than the $\Gamma$ point, and Sn further weakens it by $\sim 25 \%$ (see Table II). On the other hand, there are four $1 / 4 X_{\text {orig }}$ points in the $x$ - and $y$ directions, and two additional in energetic vicinity in the $z$ direction, which provide simply more recombination channels.

Figure 9 shows the effects of adding an acceptor concentration of $2.5 \times 10^{19} \mathrm{~cm}^{-3}$ to the emission of the system with Sn-Ge split-[110] interstitials. The effects are shown for exciton densities of $2.5 \times 10^{19} \mathrm{~cm}^{-3}$ [Fig. 9(a)] and $5.0 \times$ $10^{18} \mathrm{~cm}^{-3}$ [Fig. 9(b)]. As expected, the doping increases the emission dramatically, achieving a similar, but still weaker, emission as the Ge-Ge complex (shown in dashed lines). To achieve a stronger emission than the Ge-Ge split-[110] self-interstitial by way of the larger oscillator strength would, however, require a total suppression of the indirect valence band. The green curve shows the contribution from the transition from the $\Gamma-L$ state to the top of the valence band, which is enhanced by $p$-type doping and provides most of the emission at longer wavelengths in this condition.

The effects of $n$-type doping on the emission from a $\mathrm{Ge}$ crystal containing Sn-Ge split-[110] interstitials are shown in Fig. 10. While the enhancement due to the addition of $n$-type doping is substantial, it does not reach emission yields comparable to the Ge-Ge split-[110] self-interstitial. As shown by the green curve in Fig. 10, the emission from the $1 / 4 X_{\text {orig }}$ direct transition [ $\Delta$ transition in Figs. 10(a) and $10(b)]$ is greatly enhanced by $n$-type doping, where the effect is naturally most prominent at low exciton density [Fig 10(b)]. Due to the larger energy gap at $1 / 4 X_{\text {orig }}$ than at $\Gamma$, the emission is at shorter wavelengths than in the Ge-Ge interstitial.

Combined with compressive strain, this channel may be an avenue to improve light emission in this material by confining all electrons in the interstitial site, as discussed above. We will explore the potential of this effect in a future publication.

\section{Influence of quantum confinement}

Based on these results obtained for bulk Ge, we want to evaluate the influence of the Ge-Ge split-[110] interstitial on quantum-confined Ge structures. This is of particular importance for light emission and laser applications as threedimensionally confined quantum-dot structures have distinct advantages as laser gain material compared to bulk material, especially in association with temperature stability and lasing threshold current [41]. Along this line, for defect-enhanced quantum dots (DEQDs) that consist of compressively strained Ge on Si quantum dots containing Ge-Ge split-[110] interstitials, evidence for optically pumped lasing and the operation of light-emitting diodes at $100^{\circ} \mathrm{C}$ were reported $[10,15,16]$.

To theoretically extract the PL emission spectra from quantum-confined Ge including Ge-Ge split-[110] interstitials, we used the "truncated crystal approximation" [42] in which the zero-dimensional levels are evaluated at $E_{n}\left(\boldsymbol{k}_{i, j, k}\right)$ at discretized wave vectors $\boldsymbol{k}_{i, j, k}=\left(i \frac{\pi}{L_{x}}, j \frac{\pi}{L_{y}}, k \frac{\pi}{L_{z}}\right)$ corresponding to the dimensions of the quantum dot, with $i, j, k=$ $1,2 \ldots$ The dimensions of the hard wall box were chosen similar to those of the Ge on Si quantum dots in Ref. [10], i.e., $3 \times 20 \times 20 \mathrm{~nm}^{3}$. The main effect that we want to show here is the continuous shift of the PL emission to higher energies, shown in Fig. 11. 

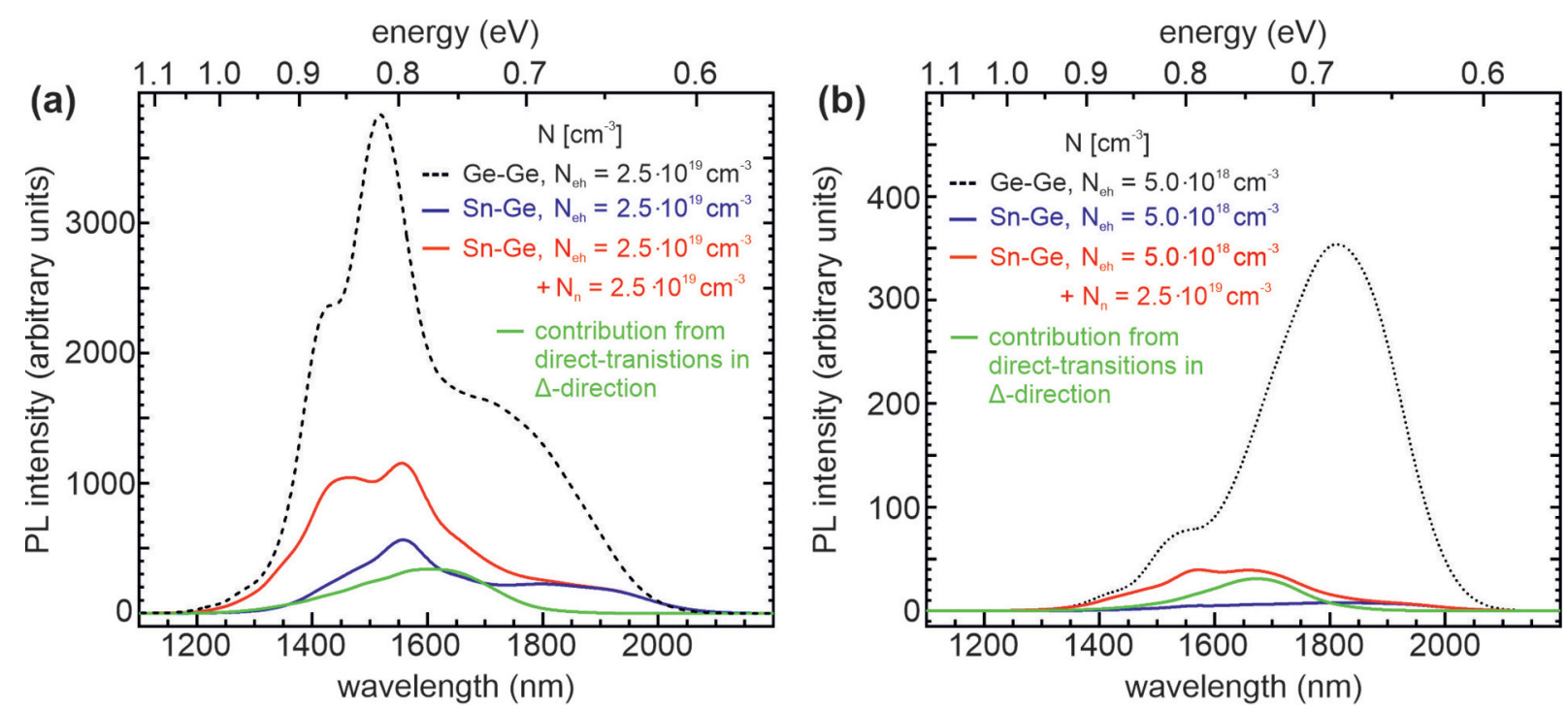

FIG. 10. (a) Calculated PL emission from Sn-Ge split-[110] interstitial at an exciton density of $2.5 \times 10^{19} \mathrm{~cm}^{-3}$ without (blue) and with additional $n$-type doping density of $2.5 \times 10^{19} \mathrm{~cm}^{-3}$ (red). The PL response from a Ge-Ge split-[110] self-interstitial without doping is depicted in black. The green curve shows the contribution from the top of the valence band at $1 / 4 X_{\text {orig }}$ to the first conduction band, also at $1 / 4 X_{\text {orig }}$, to the overall PL with doping. The contribution from the $1 / 4 X_{\text {orig }}$ bands without doping is close to zero. (b) Same case as (a) with exciton concentration $N_{\text {eh }}=5 \times 10^{18} \mathrm{~cm}^{-3}$.

While the applied quantum confinement does shift the PL emission to shorter wavelengths (Fig. 11) similar to those experimentally observed in quantum-confined Ge on Si DEQDs $[10,15,39]$, we need to stress that a quantitative comparison between these two systems is at present not feasible for three reasons.

First, the truncated crystal approximation does not consider the band offsets between Ge and Si. Thus, in this case, we can expect the quantum confinement of the valence-band state

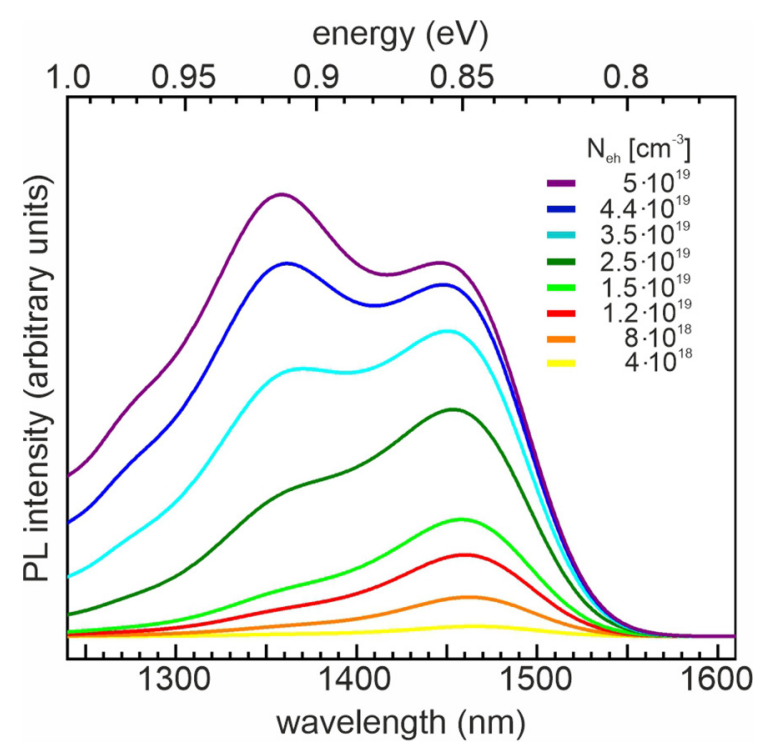

FIG. 11. Calculated photoluminescence of Ge with a Ge-Ge split-[110] self-interstitial at a different number of excitons that are quantum confined in a $3 \times 20 \times 20 \mathrm{~nm}^{3}$ box. The peak at longer wavelength is due to the $L-\Gamma$ mixed band. The $\Gamma_{\mathrm{cb}}$ band in pure Ge to the top valence bands forms the peak at shorter wavelengths. to be close to the actual one. The confinement of the conduction band will be somewhat overestimated, especially for the higher energies. Second, a further effect not considered in this work is strain produced by the inherent lattice mismatch between $\mathrm{Ge}$ and $\mathrm{Si}$. Compressive biaxial strain, as present in $\mathrm{Ge}$ on Si QDs, will push the band edge to lower energies or longer wavelengths. Examination of the detailed influence of strain (tensile and compressive) on the split interstitial in $\mathrm{Ge}$ with and without quantum confinement will, however, vastly exceed the frame of this work and will be published in an upcoming publication. The third factor that impedes comparison of the present theoretical work to DEQD light emitters $[10,15,43]$ is that for Ge on Si QDs, an intermixing of the deposited Ge with the surrounding Si matrix cannot be fully prevented. While for DEQDs examined in Refs. $[10,15,16,38]$ the low growth temperature for $\mathrm{Ge}$ of $500{ }^{\circ} \mathrm{C}$ inhibits efficient $\mathrm{Si}-G e$ alloying, it cannot be excluded that up to $10 \%$ of $\mathrm{Si}$ is present in the QDs. For QDs grown at higher temperatures or for QDs formed upon deposition of a SiGe alloy on a Si substrate, the Si concentration can be even significantly higher. Thus, in the future, we will also need to assess the combined influence of strain and SiGe alloying on the light-emission properties of bulk and quantum-confined group-IV material containing split-[110]interstitials.

Nevertheless, we can still envision possibilities for experimental realization of the nanostructures calculated in this work, i.e., quantum-confined but unstrained Ge containing split-[110] interstitial. Strain-free Ge quantum dots can, e.g., be fabricated using selective molecular-beam epitaxy on Si nanotip patterned substrates [44] or Ge nanocrystal formation through dewetting from prepatterned thin films [45]. These nanostructures can then be postformation treated by low-energy $\mathrm{Ge}$ ion implantation to achieve the optical benefits of defect-enhanced strain-free QDs described in this work. 


\section{CONCLUSIONS}

We have shown from first-principle calculations that the insertion of split-[110] interstitial defects into a Ge crystal leads to additional light-emission features not present in undisturbed Ge crystals. The emission mainly originates from three conduction bands that optically couple to the valence bands: (i) hybridized $L$ and $\Gamma$ bands in Ge due to the presence of the defect, (ii) the original $\Gamma$ band in $\mathrm{Ge}$, and to a much lesser degree (iii) a semilocalized band in the $\Delta$ line in the $\Gamma-X$ direction that directly couples to a $\Delta$ band in the valence band. Adding quantum confinement to the crystal allows for convenient tuning of the emission wavelengths across the telecommunication wavelength range from 1300 to $1900 \mathrm{~nm}$. Thereby, an increased number of defect centers can lead to emission quenching since the original $\Gamma$-like conduction band in Ge becomes a mixed state, losing its direct-band character

Introduction of a Ge-Sn split-[110] interstitial to Ge doubles the oscillator strength of the involved $\Gamma-L$ transitions but moves the top of the valence band away from the $\Gamma$ point to $1 / 4 X$ in the Brillouin zone. Consequently, the effective light emission decreases due to the resulting indirect band gap compared to the Ge-Ge split-[110] self-interstitial, which induces a direct band gap. $n$ - and $p$-type doping is a valuable tool to significantly enhance the light emission by populating band extrema featuring direct character and finite oscillator strengths at either the conduction or valence band. Thus, we expect that the opening of direct recombination paths in Ge by inserting split-[110] interstitials will be of particular interest regarding applications in future Si photonics devices.

\section{ACKNOWLEDGMENTS}

This work was funded by the Austrian Science Fund (FWF): Grants No. Y1238-N36 and No. P30564-NBL, the Linz Institute of Technology (LIT): Grant No. LIT-2019-7SEE-114, and Science Foundation Ireland (SFI) Grant No. 19/FFP/6953. Funding was also provided by the EU Grant H2020 QuantERA ERA-NET via the Quantum Technologies project CUSPIDOR, which is co-funded by FWF (Grant No. I3760) and SFI (Grant No. 17/QERA/3473). P.S. is Brno Ph.D. Talent Scholarship Holder-Funded by the Brno City Municipality. His work was financed by the project CUSPIDOR which has received funding from the QuantERA ERA-NET Cofund in Quantum Technologies implemented within the European Union's Horizon 2020 Programme. In addition, this project has received national funding from the Ministry of Education, Youth and Sports of the Czech Republic and funding from European Union's Horizon 2020 (2014-2020) research and innovation framework programme under Grant Agreement No. 731473. P.B. acknowledges the Vienna Scientific Cluster (VSC), where part of the calculations have been performed. We would like to thank Petr Klenovsky for his support and interesting discussions.
[1] J. Yang, Z. Liu, P. Jurczak, M. Tang, K. Li, S. Pan, A. Sanchez, R. Beanland, J.-C. Zhang, H. Wang, F. Liu, Z. Li, S. Shutts, P. Smowton, S. Chen, A. Seeds, and H. Liu, J. Phys. D: Appl. Phys. 54, 035103 (2021).

[2] S. Wirths, R. Geiger, N. V. Den Driesch, G. Mussler, T. Stoica, S. Mantl, Z. Ikonic, M. Luysberg, S. Chiussi, J. M. Hartmann, H. Sigg, J. Faist, D. Buca, and D. Grützmacher, Nat. Photonics 9, 88 (2015).

[3] V. Reboud, A. Gassenq, N. Pauc, J. Aubin, L. Milord, Q. M. Thai, M. Bertrand, K. Guilloy, D. Rouchon, J. Rothman, T. Zabel, F. Armand Pilon, H. Sigg, A. Chelnokov, J. M. Hartmann, and V. Calvo, Appl. Phys. Lett. 111, 092101 (2017).

[4] Y. Zhou, Y. Miao, S. Ojo, H. Tran, G. Abernathy, J. M. Grant, S. Amoah, G. Salamo, W. Du, J. Liu, J. Margetis, J. Tolle, Y.-H. Zhang, G. Sun, R. A. Soref, B. Li, and S.-Q. Yu, Optica 7, 924 (2020).

[5] M. J. Süess, R. Geiger, R. A. Minamisawa, G. Schiefler, J. Frigerio, D. Chrastina, G. Isella, R. Spolenak, J. Faist, and H. Sigg, Nat. Photonics 7, 466 (2013).

[6] F. T. Armand Pilon, A. Lyasota, Y.-M. Niquet, V. Reboud, V. Calvo, N. Pauc, J. Widiez, C. Bonzon, J. M. Hartmann, A. Chelnokov, J. Faist, and H. Sigg, Nat. Commun. 10, 2724 (2019).

[7] A. Gassenq, K. Guilloy, N. Pauc, J.-M. Hartmann, G. O. Dias, D. Rouchon, S. Tardif, J. Escalante, I. Duchemin, Y.-M. Niquet, A. Chelnokov, V. Reboud, and V. Calvo, Thin Solid Films 613, 64 (2016).
[8] M. Virgilio, C. L. Manganelli, G. Grosso, G. Pizzi, and G. Capellini, Phys. Rev. B 87, 235313 (2013).

[9] D. Saladukha, M. B. Clavel, F. Murphy-Armando, G. GreeneDiniz, M. Grüning, M. K. Hudait, and T. J. Ochalski, Phys. Rev. B 97, 195304 (2018).

[10] M. Grydlik, F. Hackl, H. Groiss, M. Glaser, A. Halilovic, T. Fromherz, W. Jantsch, F. Schäffler, and M. Brehm, ACS Photonics 3, 298 (2016).

[11] C. L. Xiong, B. Bell, and B. J. Eggleton, Nanophotonics 5, 427 (2016).

[12] E. Murray, D. J. P. Ellis, T. Meany, F. F. Floether, J. P. Lee, J. P.Griflths, G. A. C. Jones, I. Farrer, D. A. Ritchie, A. J. Bennett, and A. J. Shields, Appl. Phys. Lett. 107, 171108 (2015).

[13] M. D. Moreira, R. H. Miwa, and P. Venezuela, Phys. Rev. B 70, 115215 (2004).

[14] A. J. R. da Silva, A. Janotti, A. Fazzio, R. J. Baierle, and R. Mota, Phys. Rev. B 62, 9903 (2000).

[15] M. Grydlik, M. T. Lusk, F. Hackl, A. Polimeni, T. Fromherz, W. Jantsch, F. Schäffler, and M. Brehm, Nano Lett. 16, 6802 (2016).

[16] P. Rauter, L. Spindlberger, F. Schäffler, T. Fromherz, J. Freund, and M. Brehm, ACS Photonics 5, 431 (2018).

[17] P. Blaha, K. Schwarz, F. Tran, R. Laskowski, G. K. H. Madsen, and L. D. Marks, J. Chem. Phys. 152, 074101 (2020).

[18] P. Blaha, K. Schwarz, G. K. H. Madsen, D. Kvasnicka, J. Luitz, R. Laskowski, F. Tran, and L. D. Marks, WIEN2k: An Augmented Plane Wave plus Local Orbitals Program for Calculating 
Crystal Properties (Vienna University of Technology, Austria, 2018).

[19] W. Kohn and L. J. Sham, Phys. Rev. 140, A1133 (1965).

[20] J. P. Perdew and Y. Wang, Phys. Rev. B 45, 13244 (1992).

[21] J. P. Perdew, K. Burke, and M. Ernzerhof, Phys. Rev. Lett. 77, 3865 (1996); 78, 1396(E) (1997).

[22] F. Tran and P. Blaha, Phys. Rev. Lett. 102, 226401 (2009).

[23] P. Borlido, T. Aull, A. W. Huran, F. Tran, M. A. L. Marques, and S. Botti, J. Chem. Theory Comput. 15, 5069 (2019).

[24] F. Tran, J. Doumont, L. Kalantari, A. W. Huran, M. A. L. Marques, and P. Blaha, J. Appl. Phys. 126, 110902 (2019).

[25] O. Rubel, A. Bokhanchuk, S. J. Ahmed, and E. Assmann, Phys. Rev. B 90, 115202 (2014).

[26] https://github.com/rubel75/fold2Bloch-Wien2k

[27] X. Gonze, F. Jollet, F. Abreu Araujo, D. Adams, B. Amadon, T. Applencourt, C. Audouze, J.-M. Beuken, J. Bieder, A. Bokhanchuk, E. Bousquet, F. Bruneval, D. Caliste, M. Cote, F. Dahm, F. Da Pieve, M. Delaveau, M. Di Gennaro, B. Dorado, C. Espejo, G. Geneste, L. Genovese, A. Gerossier, M. Giantomassi, Y. Gillet, D. R. Hamann, L. He, G. Jomard, J. Laflamme Janssen, S. Le Roux, A. Levitt, A. Lherbier, F. Liu, I. Lukacevic, A. Martin, C. Martins, M. J. T. Oliveira, S. Ponce, Y. Pouillon, T. Rangel, G.-M. Rignanese, A. H. Romero, B. Rousseau, O. Rubel, A. A. Shukri, M. Stankovski, M. Torrent, M. J. Van Setten, B. Van Troeye, M. J. Verstraete, D. Waroquier, J. Wiktor, B. Xue, A. Zhou, and J. W. Zwanziger. Comput. Phys. Commun. 205, 106 (2016).

[28] C. Hartwigsen, S. Goedecker, and J. Hutter, Phys. Rev. B 58, 3641 (1998).

[29] F. Murphy-Armando and S. Fahy, Phys. Rev. Lett. 97, 096606 (2006).

[30] F. Murphy-Armando and S. Fahy, Phys. Rev. B 78, 035202 (2008).
[31] W. Schafer and M. Wegener, Semiconductor Optics and Transport Phenomena (Springer, Berlin, 2002), p.363.

[32] W. W. Chow, Appl. Phys. Lett. 100, 191113 (2012).

[33] J. Menéndez, C. D. Poweleit, and S. E. Tilton, Phys. Rev. B 101, 195204 (2020).

[34] S. M. O’Mahony, F. Murphy-Armando, É. D. Murray, J. D. Querales-Flores, I. Savić, and S. Fahy, Phys. Rev. Lett. 123, 087401 (2019).

[35] F. Murphy-Armando, É. D. Murray, I. Savić, M. Trigo, D. Reis, and S. Fahy, arXiv:1911.12145v2.

[36] T. P. McLean and R. Loudon, J. Phys. Chem. Solids 13, 1 (1960).

[37] P. E. Blöchl, E. Smargiassi, R. Car, D. B. Laks, W. Andreoni, and S. T. Pantelides, Phys. Rev. Lett. 70, 2435 (1993).

[38] H. Groiss, L. Spindlberger, P. Oberhumer, F. Schäffler, T. Fromherz, M. Grydlik, and M. Brehm, Semicond. Sci. Technol. 32, 02LT01 (2017).

[39] L. Spindlberger, J. Aberl, A. Polimeni, J. Schuster, J. Hörschläger, T. Truglas, H. Groiss, F. Schäffler, T. Fromherz, and M. Brehm, Crystals 10, 351 (2020).

[40] M. El Kurdi, T. Kociniewski, T.-P. Ngo, J. Boulmer, D. Débarre, P. Boucaud, J. F. Damlencourt, O. Kermarrec, and D. Bensahel, Appl. Phys. Lett. 94, 191107 (2009).

[41] Z. I. Alferov, Rev. Mod. Phys. 73, 767 (2001).

[42] S. B. Zhang, C.-Y. Yeh, and A. Zunger, Phys. Rev. B 48, 11204 (1993).

[43] L. Spindlberger, S. Prucnal, J. Aberl, and M. Brehm, Phys. Status Solidi A 216, 1900307 (2019).

[44] G. Niu, G. Capellini, M. A. Schubert, T. Niermann, P. Zaumseil, J. Katzer, H.-M. Krause, O. Skibitzki, M. Lehmann, Y.-H. Xie, H. Von Känel, and T. Schroeder, Sci. Rep. 6, 22709 (2016).

[45] I. Berbezier, M. Aouassa, A. Ronda, L. Favre, M. Bollani, R. Sordan, A. Delobbe, and P. Sudraud, J. Appl. Phys. 113, 064908 (2013). 